\title{
Transport in organic semiconductors in large electric fields: From thermal activation to field emission
}

\author{
J. H. Worne ${ }^{1}$, J. E. Anthony ${ }^{2}$, D. Natelson ${ }^{1,31,2,3}$ \\ 1) Department of Electrical and Computer Engineering, Rice University, 6100 Main St., Houston, \\ $T X 77005$ \\ ${ }^{2)}$ Department of Chemistry, University of Kentucky, Lexington, KY 40506-0055 \\ 3) Department of Physics and Astronomy, Rice University, 6100 Main St., Houston, \\ TX 77005
}

Understanding charge transport in organic semiconductors in the presence of large electric fields is relevant to many potential applications. Here we present transport measurements in organic field-effect transistors based on poly(3-hexylthiophene) (P3HT) and 6,13-bis(triisopropyl-silylethynyl) pentacene (TIPS-pentacene) with short channels, from room temperature down to $4.2 \mathrm{~K}$. Near $300 \mathrm{~K}$ transport in both systems is well described by a model of thermally assisted hopping with Poole-Frenkel-like electric field enhancement of the mobility. At low temperatures and large gate voltages, transport in both materials becomes nearly temperature independent, crossing over into a regime described by field-driven tunneling. These data, particularly in TIPS-pentacene, show that great caution must be exercised when considering more exotic models of low- $T$ transport in these materials.

Charge transport in organic semiconductors in the presence of large electric fields is important in organic light-emitting diodes (OLEDs), the channels of organic field-effect transistors (OFETs), and organic photovoltaic devices. Much recent work has focused on the effects of large electric fields on the injection process ${ }^{1-3}$ as well as on transport within the semiconductor bulk ${ }^{2,4-8}$. In general, this is a complex, nonequilibrium problem, involving charge carriers with moderately strong couplings to vibrational degrees of freedom, and a disordered environment resulting in localization and a strongly energy dependent density of states. As a result, transport characteristics, usually parametrized by a mobility, $\mu$, depend nontrivially on temperature, electric field, and carrier density $\underline{6,7,9,10}$.

Near room temperature, the field dependence of the mobility is often reasonably described (over a limited temperature and field range) by an effective PooleFrenkel (PF) model of mobility ${ }^{11}$. In this model ${ }^{11}$, mobility $\mu_{\mathrm{PF}} \propto \mu_{0}(T) \exp (\gamma \sqrt{E})$, where $\mu_{0}$ is the zero-field mobility and $E$ is the electric field. Both $\mu_{0}$ and $\gamma$ vary like $1 / T$ in this limited range $\underline{4,5,8}$. In the Poole-Frenkel regime, one can think of the field dependence arising from field-induced distortion of the disorder potential of the localized carrier states.

At lower temperatures and strong source-drain fields, it has been noted ${ }^{5}$ that conduction in OFETs is highly nonlinear and approaches a temperature-independent regime as $T \rightarrow 0$. The physical mechanism for this temperature independence is in dispute. Recent work done by Dhoot, et al. ${ }^{12}$ argued that the crossover to temperature-independent nonlinear conduction at low temperatures and large gate voltages was a signature of a voltage-driven insulator-to-metal transition. Prigodin and Epstein ${ }^{13}$ argue instead that what is observed is a field-induced crossover from thermal activation to a field emission hopping regime ${ }^{14}$ previously examined by Shklovskii ${ }^{15}$. This point of view is ex- tended by Wei et al $\stackrel{16}{n}$, who find that a multistep tunneling model explains the observed strong dependences on gate and source-drain voltage. More recently, Yuen et $a l^{17}$ have looked at the evolution of carrier transport from $300 \mathrm{~K}$ to $4 \mathrm{~K}$ and below, and claim further evidence for an insulator-to-metal transition in devices based on the and poly(2,5-bis(3-tetradecylthiophen-2yl)thieno[3,2-b]thiophene) (PBTTT). Specifically, they contend that their data are well described by a Tomonaga-Luttinger Liquid (TLL) model of transport originally developed for truly one-dimensional metals.

To address this controversial issue, we performed transport experiments from room temperature down to cryogenic temperatures in short-channel bottomcontact OFET devices based on two different molecules, P3HT ${ }^{18}$ and 6,13-bis(triisopropyl-silylethynyl) (TIPS)pentacene ${ }^{19}$. P3HT is a polymer semiconductor that tends toward glassy or nanocrystalline structure, while TIPS-pentacene is a solution-processable small molecule that forms van der Waals bonded molecular crystals. As the temperature is decreased, we observe that charge carrier behavior evolves from Poole-Frenkel-like activated hopping at high temperatures to temperatureindependent hopping consistent with field emission at low temperatures in both P3HT and TIPS-pentacene systems. While the TLL analysis approach ${ }^{17,20}$ produces compelling plots, we argue that this is fortuitous, particularly since there is no reason to expect TLL physics to be relevant in the TIPS-pentacene case.

We used degenerately doped p-type silicon with $200 \mathrm{~nm}$ of thermally grown oxide, which serves both as the substrate and the gate in our experiments. Platinum electrodes were fabricated using standard electron beam lithography, electron beam evaporation and liftoff processing with channel widths, $W$, of $50 \mu \mathrm{m}$ and channel length, $L$, of $300 \mathrm{~nm}$ (device A) and $W=200 \mu \mathrm{m}$ and $L=1 \mu \mathrm{m}$ (device B). On both substrates other electrodes of fixed width and varying channel lengths were prepared for transmission line estimates of the contact 
resistance. Samples were rinsed using isopropanol and acetone followed by an oxygen plasma cleaning for two minutes. Samples were then spin-coated with hexamethyldisilazane (HMDS) at 3000 RPM for 30 seconds, followed by a bake at $130 \mathrm{C}$ for 20 minutes.

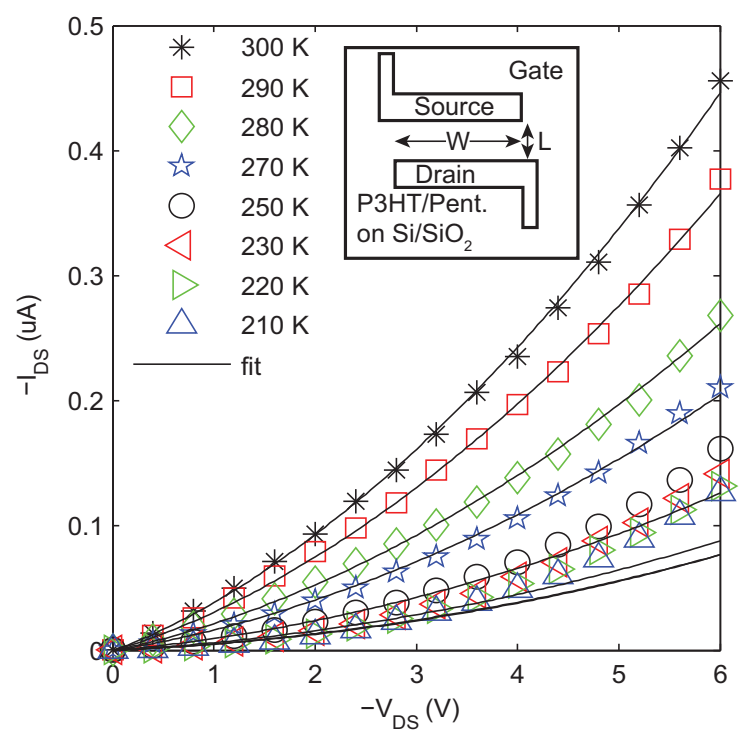

FIG. 1. $I_{\mathrm{D}}-V_{\mathrm{DS}}$ curves for device A over a $100 \mathrm{~K}$ temperature range at $V_{g}=-80 \mathrm{~V}$. Fit lines are generated from a PooleFrenkel-like field dependence of the mobility, as explained in the text. The deviation from theory as $\mathrm{T}$ decreases indicates the beginning of the crossover from activated hopping into field emission.

P3HT was spin-cast from chloroform at a $0.1 \%$ by weight concentration onto device A and TIPS-pentacene was drop cast from toluene at a $1 \%$ by weight concentration onto device B. Samples were measured in a variable temperature probe station with base pressure of $1 \times 10^{-6}$ Torr. Transmission line measurements on the cofabricated device arrays were made on chip to measure device mobilities, which were $4.6 \times 10^{-2} \mathrm{~cm}^{2} / \mathrm{Vs}$ (P3HT) and $1.1 \times 10^{-4} \mathrm{~cm}^{2} / \mathrm{Vs}$ (TIPS-pentacene), respectively. We then extracted contact resistances for our devices and determined that they are much smaller than the resistance within the device channel, indicating these devices are bulk dominated. As shown in previous work ${ }^{21}$, as the temperature is reduced, bulk channel resistance increases more rapidly than the contact resistance, so that our measurements remain bulk limited down to low temperatures. Measurement source-drain voltages $V_{\mathrm{DS}}$ were set so that the average source-drain electric fields in the channels of both device $\mathrm{A}$ and $\mathrm{B}$ were similar, with a maximum electric field of $20 \mathrm{MV} / \mathrm{m}$ in device $\mathrm{A}$ and $10 \mathrm{MV} / \mathrm{m}$ in device $\mathrm{B}$.

We confirmed that our P3HT sample follows PF behavior at high $T$, as shown in figure 1. The inset of figure 1 is a cartoon describing our device geometry. The data shown here are at one particular gate voltage, $V_{\mathrm{G}}$, at a variety of temperatures. At each temperature data for all gate voltages are analyzed $\underline{8}$ using the form

$$
I_{\mathrm{D}}=\frac{\mu_{0} w C_{i}}{L} \exp \left(\gamma \sqrt{V_{\mathrm{DS}} / L}\right)\left[\left(V_{\mathrm{G}}-V_{\mathrm{T}}\right) V_{\mathrm{DS}}-\frac{V_{\mathrm{DS}}^{2}}{2}\right]
$$

where $\mu_{0}$ is the (gate- and temperature-dependent) zerofield mobility, $C_{i}$ is the capacitance per area of the gate oxide, $\gamma$ is a prefactor that is found to vary like $1 / T$, and $V_{\mathrm{T}}$ is the threshold voltage. As $T$ decreases, we note a deviation of the data from the PF model. This will be addressed below. Analysis of the TIPS-pentacene data is qualitatively identical.

We tried plotting our data in the manner suggested by the TLL analysis $\frac{17,20}{10}$. The expression for the current in the TLL picture is $17,22,23$

$$
I=I_{0} T^{\alpha+1} \sinh \left(\gamma^{\prime} e V / k_{\mathrm{B}} T\right)\left|\Gamma\left((1+\beta) / 2+i V / \pi k_{\mathrm{B}} T\right)\right|^{2},
$$

where $\Gamma$ is the Gamma function, $\alpha$ and $\beta$ are phenomenological exponents estimated from plots of conductance vs. $T$ and $I(V)$, respectively, and $\gamma^{\prime}$ is a phenomenological parameter thought to be related to the amount of disorder (tunneling barriers) along the $1 \mathrm{~d}$ structure. Based on this equation, the idea is (at fixed $V_{\mathrm{G}}$ ) to plot $I_{\mathrm{D}} / T^{\alpha+1}$ vs. $\left(e V_{\mathrm{DS}} / k_{\mathrm{B}} T\right)$, where $\alpha$ is a fit parameter. If a particular value of $\alpha$ collapses all of the data over the whole temperature range onto a single curve, it is tempting to conclude that the TLL picture is valid. Critical to the TLL theory validity is that the system in question be one-dimensional. In polymers such as PBTTT and $\mathrm{P} 3 \mathrm{HT}$, carrier mobility along the polymer chain is generally much higher than between chains, implying that they may be relevant.

We present the same style of analysis in Figure 2, with the P3HT data shown in A and the TIPS-pentacene data shown in B. We are able to collapse our data onto a single line as $T$ is decreased, with choices for $\alpha(5.43$ for P3HT, 7.1 for TIPS-pentacene) that are not wildly different from those reported ${ }^{17}$ for $\operatorname{PBTTT}(5.4,4.3)$ or polyaniline fibers ${ }^{20}$ (5.5). Recall that TIPS-pentacene is a short chain molecule, without the mobility anisotropy found in PBTTT or P3HT. It seems extremely unlikely that TIPS-pentacene can be described by TLL theory for a one-dimensional metal, despite the apparent collapse of its $I_{\mathrm{D}}-V_{\mathrm{DS}}$ curves onto a single master line.

With the freedom to adjust $\alpha$, plotting scaled data as in Fig. 2 becomes unwise. As $T$ decreases, both P3HT and TIPS-pentacene $I_{\mathrm{D}}-V_{\mathrm{DS}}$ curves become increasingly non-linear and temperature independent. Plotting the data of Figure 2 turns roughly power law trends over a limited voltage range into a linear segment on such a log-log plot. The freedom to choose $\alpha$ while plotting allows fine-tuning of the subsequent temperature curves to lie on the same line. Because current and voltage are plotted as $I_{\mathrm{D}} / T^{\alpha+1}$ and $e V_{\mathrm{DS}} / k_{\mathrm{B}} T$, decreasing $T$ moves subsequent temperature data sets up and to the right on the graph, even if the data themselves do not change with temperature at all. Data collapse with this plotting 


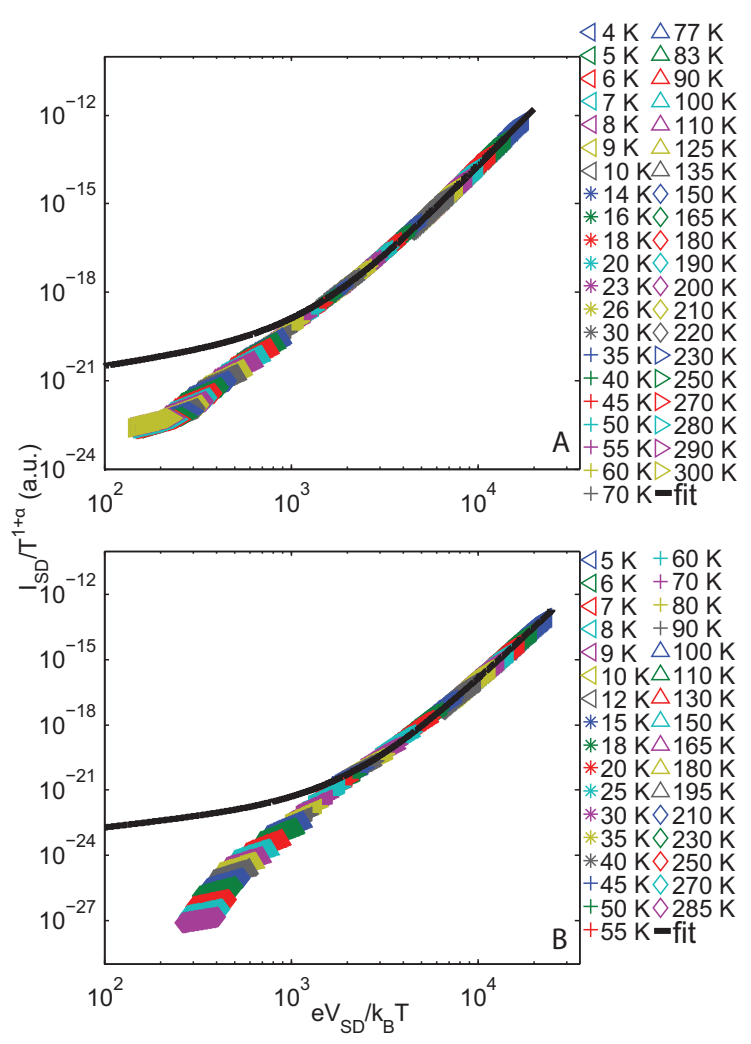

FIG. 2. Plotting $I_{\mathrm{D}}$ vs. $V_{\mathrm{DS}}$ in scaled coordinates as suggested by Eq. (2). Top data is on device A (P3HT, $\left.V_{\mathrm{G}}=-80 \mathrm{~V}\right)$, while bottom data is on device $\mathrm{B}$ (TIPS-pentacene, $V_{\mathrm{G}}=$ $-70 \mathrm{~V}$ ). Solid lines are fits to Eq. (2). For device A, $\alpha=$ $5.43, \gamma^{\prime}=4 \times 10^{-3}$; for device B, $\alpha=7.1, \gamma^{\prime}=3 \times 10^{-3}$; for both fits, we used the theoretical expectation $\beta=\alpha+$ 1. As explained in the text, the apparent scaling collapse is fortuitous, rather than the result of Tomonaga-Luttinger Liquid physics.

procedure is not sufficient to demonstrate TLL physics.

Instead, as $T$ decreases, we propose that carrier transport evolves from activation hopping into field emission hopping 13,16 , where $\mu_{P F}$ becomes $\mu_{F E} \propto$ $\mu_{0} \exp \left(-\sqrt{E_{0} / E}\right)$ in an equation analogous to Eq. (1). Here $E=V_{\mathrm{DS}} / L$ is the average electric field within the channel, while $E_{0}\left(V_{\mathrm{G}}\right)$ is temperature independent and is expected 13 to depend on the disorder of the sample. We plot the data for our lowest temperature $I_{\mathrm{D}}-V_{\mathrm{DS}}$ curves in figure 3 and note that near this temperature our data are well fit by a temperature-independent $\mu_{0}$. A detailed comparison to the multiple tunneling mode $\mathrm{l}^{16}$ will require further extensive data, particularly examining the effects of gate voltage on $E_{0}$ and attempting to analyze the crossover regime of temperature and voltage.

In conclusion, we present data that illustrates both the high and low temperature transport behavior of two chemically unique organic semiconductors. The data are consistent with a crossover from Poole-Frenkellike activated hopping near room temperature to a

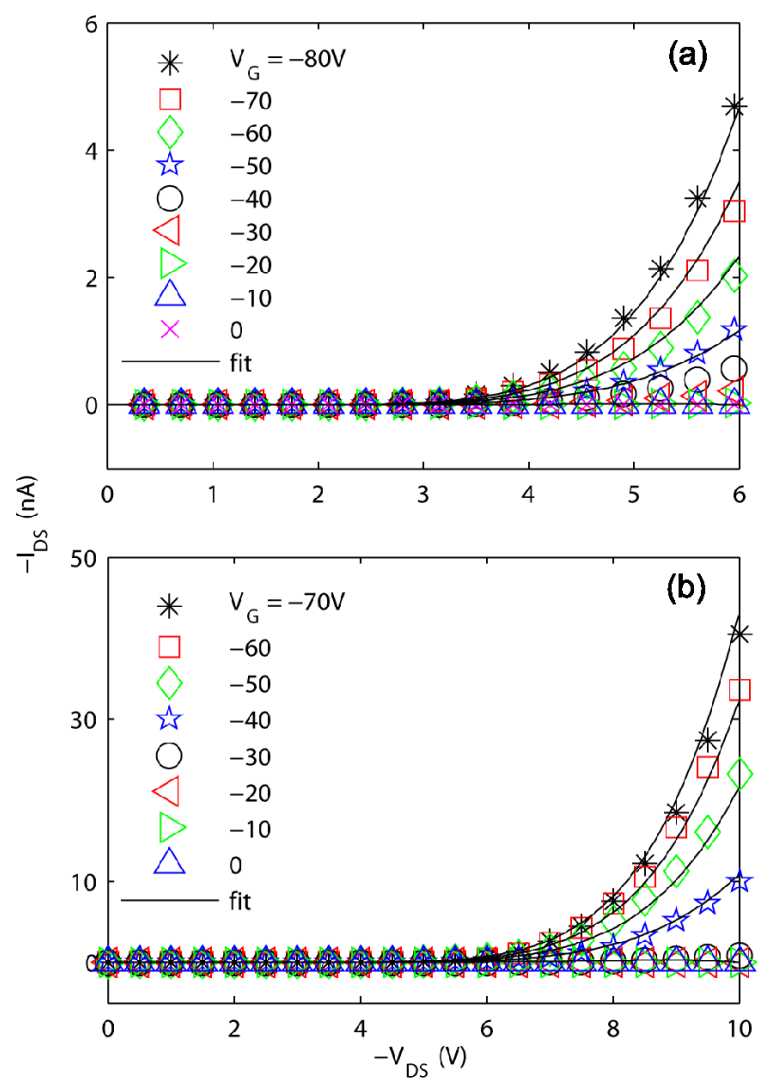

FIG. 3. Top is P3HT (device A) measured at various gate voltages at $4.2 \mathrm{~K}$; bottom is TIPS-pentacene (device B) measured at $5 \mathrm{~K}$. Black lines are fits using the field emission hopping model expression for mobility, $\mu \propto \exp \left(-\left(E_{0} / E\right)^{1 / 2}\right)$.

temperature-independent field emission hopping process $\left(\sim \exp \left(-\sqrt{E_{0} / E}\right)\right)$ at cryogenic temperatures. We further find that a scaling approach is insufficient to test for Tomonaga-Luttinger Liquid physics, as it shows apparent TLL consistency even in TIPS-pentacene, a material that has no microscopic basis for TLL physics. Further detailed investigations should be able to test sophisticated models for the full temperature and voltage dependence of transport in these rich material systems.

${ }^{1}$ J. C. Scott, J. Vac. Sci. Tech. A 21, 521 (2003).

${ }^{2}$ T. N. Ng, W. R. Silveira, and J. A. Marohn, Phys. Rev. Lett. 98, 1 (2007).

${ }^{3}$ S. Scheinert and G. Paasch, J. Appl. Phys. 105 (2009).

${ }^{4}$ P. W. M. Blom, M. J. M. De Jong, and M. G. Van Munster, Phys. Rev. B 55, R656 (1997).

${ }^{5}$ B. H. Hamadani and D. Natelson, J. Appl. Phys. 95, 1227 (2004).

${ }^{6}$ W. F. Pasveer et al., Phys. Rev. Lett. 94, 206601 (2005).

${ }^{7}$ B. N. Limketkai, P. Jadhav, and M. A. Baldo, Phys. Rev. B 75, 113203 (2007).

${ }^{8}$ B. H. Hamadani et al., J. Appl. Phys. 102, 044503 (2007).

${ }^{9}$ C. Tanase, E. J. Meijer, P. W. M. Blom, and D. M. de Leeuw, Phys. Rev. Lett. 91, 216601 (2003).

${ }^{10}$ N. Tessler, Y. Preezant, N. Rappaport, and Y. Roichman, Adv. Mater. 21, 2741 (2009).

${ }^{11}$ J. Frenkel, Phys. Rev. 54, 647 (1938). 
${ }^{12}$ A. Dhoot, G. Wang, D. Moses, and A. Heeger, Phys. Rev. Lett. 96, 246403 (2006).

${ }^{13}$ V. N. Prigodin and A. J. Epstein, Phys. Rev. Lett. 98, 259703 (2007).

${ }^{14}$ A. Larkin and D. E. Khmelnitski, Sov. Phys. JETP 56, 647 (1982).

${ }^{15}$ B. I. Shklovskii, Sov. Phys. - Semicond. 6, 1964 (1973).

${ }^{16}$ J. H. Wei, Y. L. Gao, and X. R. Wang, Appl. Phys. Lett. 94, 073301 (2009).
${ }^{17}$ J. D. Yuen et al., Nature Materials 8, 572 (2009).

${ }^{18}$ R. D. McCullough, R. D. Lowe, M. Jayaraman, and D. L. Anderson, J. Org. Chem. 58, 904 (1993).

${ }^{19}$ S. K. Park, T. N. Jackson, J. E. Anthony, and D. A. Mourey, Appl. Phys. Lett. 91, 063514 (2007).

${ }^{20}$ A. Aleshin, Adv. Mater. 18, 17 (2006).

${ }^{21}$ B. H. Hamadani and D. Natelson, Appl. Phys. Lett. 84, 443 (2004).

${ }^{22}$ L. Balents, cond-mat/9906032 (1999).

${ }^{23}$ M. Bockrath et al., Nature 397, 598 (1999). 